\title{
Imaging the patient with a new diagnosis of heart failure in the contemporary era
}

\author{
James E. Udelson, $\mathrm{MD}^{\mathrm{a}}$ \\ a Division of Cardiology, The CardioVascular Center, Tufts Medical Center, Boston, MA \\ Received Jun 22, 2015; accepted Jun 22, 2015 \\ doi: 10.1007/s12350-015-0222-4
}

The case study presented by Dr. Iskandrian describes a fairly typical scenario that we often see in practice - a patient with a new diagnosis of heart failure (HF) and systolic dysfunction. Consistent with existing Guidelines, the initial test to begin to parse the various potential etiologies is a transthoracic echocardiogram. ${ }^{1}$ From that imaging study in this case, we learn about the systolic dysfunction, but importantly the results have taken valvular disease off the table. We are now challenged by Dr. Iskandrian to move to the important next step in the evaluation, to differentiate ischemic from nonischemic cardiomyopathy.

From our imaging perspective, there are two separate but related questions to be answered: first, is coronary artery disease (CAD) present and is it the cause of the cardiomyopathy? Second, if CAD is present, is revascularization an option that will benefit the patient?

Before commenting on the cases made for the individual modalities by the authors, it is worth taking a moment to frame how we need to think about testing in the contemporary era. For the initial few decades during the development of our noninvasive imaging techniques, we have had the luxury of needing to use little restraint in ordering tests and gathering information, allowing comprehensive understanding of the prognostic value of the tests and other features of clinical interest, and driving robust published literature.

However, in recent years and increasingly in the very near future, the changing health care delivery structures within which we do our work are forcing us to re-think that strategy. In Accountable Care Organizations (ACOs) and similar care systems, we are accountable for both good outcomes and also for the

Reprint requests: James E. Udelson, MD, Division of Cardiology, The CardioVascular Center, Tufts Medical Center, Box 70, 800 Washington St, Boston, MA 02111; judelson@tuftsmedicalcenter. org

J Nucl Cardiol 2015;22:975-9.

$1071-3581 / \$ 34.00$

Copyright (C) 2015 American Society of Nuclear Cardiology. resources used to arrive at those outcomes. So in some ways our new thinking has to be along the lines of "...how little testing can I do to have sufficient information to make a clinical decision that will result in benefit to my patient?",

From that perspective, many tests that have clear prognostic value may not have much value clinically. As a general example, consider the usefulness of serum biomarkers that are prognostic, with extensive literature supporting their risk stratification value. In the heart failure world, two such biomarkers have been approved for use relatively recently, ST2 and galectin-3. Soluble ST2 and the ST2 receptor are involved in interleukin-33based signaling in the heart and reflect activity of this stress-response system. ${ }^{2}$ Galectin-3 is a protein that has been suggested to be a link between the processes of inflammation and fibrosis. ${ }^{3}$ For both of these markers, serum levels have been prognostic in several settings of the heart failure syndrome and have shown varying degrees of incremental risk stratification value when analyzed according to current rigorous standards for biomarkers. ${ }^{3,4}$ Yet there is no clear clinical decision that emanates from knowledge of whether these markers are low or high. Patients with heart failure and established systolic dysfunction are all treated aggressively with neurohormonal antagonists, implantable defibrillators, and resynchronization devices (when indicated), across the symptomatic spectrum. ${ }^{1}$ Referral for advanced therapies such as transplant or ventricular assist devices is made on the basis of progressive symptoms and cannot be done solely on the basis of high-risk prognosis identified by a biomarker without progressive symptoms. Thus, knowledge of the biomarker in general leads to no specific decision and thus is of very limited clinical value.

This is an example of how we need to be facile switching between two hats-our "academic" hat, under which we try to learn about how new or old tests might help decisions about our patients (and hopefully someone is funding us to do that learning), and a 
"clinical" hat, under which we can really only use tests that do have clinical value for informing decision making. With all of that as background to frame our thinking, let us examine the cases made by the authors for their modalities.

Drs. Ahn and Samady make the case for comprehensive initial invasive assessment of this patient with a new diagnosis of heart failure, ${ }^{5}$ with right and left heart catheterization and coronary angiography, accompanied by consideration of numerous other contemporary invasive physiologic measures involving coronary flow reserve, fractional flow reserve (FFR), high-resolution imaging of coronary plaque and the vascular wall, and then electro-anatomic mapping for assessment of viability.

I do not think I would want them in my ACO. That is a lot of information gathering (at relatively high cost and invasive level of risk) that may not be necessary. For example, consider their simple statement that the right heart catheterization hemodynamics to measure " "...wedge pressure... would allow tuning of the patient's diuretic dosing which otherwise can be challenging both for the patient and provider.' That hemodynamic measures would enable very finely tuned medical therapy is an attractive thought and hypothesis, but one that has been tested and disproved by a randomized trial. ${ }^{6}$ It is an example of information that is expensive and invasive to obtain but is not routinely needed to manage such a patient, no matter how attractive it might be to have at hand. Assessing microvascular contribution to blunted coronary flow reserve is of pathophysiologic interest but has no clear treatment implication. Guidelines suggest that invasive coronary angiography is a class IIa (LOE C) recommendation "... when ischemia may be contributing to HF' '. So while Ahn and Samady are correct in that these techniques can indeed provide very comprehensive information, much of it is not routinely important to know in such a patient. Indeed even invasive coronary angiography can be safely avoided if noninvasive testing adequately rules out CAD, as discussed further below.

Danad and Min describe the potential use of comprehensive CT imaging. ${ }^{7}$ It is attractive in many ways. However, the assessment of tissue characterization is at this point much less mature than other modalities and not as yet widely practiced or extensively validated. So for practical purposes, CT would be providing noninvasive information on the status of the coronary arteries. If normal, that is very helpful. If not normal, additional information would be needed. CT-based assessment of FFR is an exciting development for evaluation of CAD, ${ }^{8}$ but it has not been studied in the setting of $\mathrm{HF}$ and systolic dysfunction, and its correlation with downstream tissue viability — which is what we really need to know-is unknown.
Argulian and Chaudhry ${ }^{9}$ recommend the use of stress echocardiography and techniques such as strain imaging and estimated filling pressures. Much of their discussion on the use of stress echocardiography involves assessing prognosis in more general settings than HF, which as discussed above is not necessarily by itself directly actionable. The performance of stress echocardiography for detection of CAD in the setting of baseline abnormalities of regional or global function is modest as they point out. While there is an extensive literature on the use of dobutamine echocardiography for viability assessment, ${ }^{10}$ the visually subjective nature of the analysis is a limitation. Techniques such as strain imaging, while of great interest pathophysiologically from an academic standpoint, have no clear practical implication. Finally, while some advocate that noninvasive estimation of filling pressures is robust, several papers have now challenged the validity of those measures in both an acute and more chronic HF setting. ${ }^{11,12}$ So while an initial resting echocardiogram is invaluable, the information following stress may not fully address the issues routinely at hand. Argulian and Chaudhry also suggest that in considering testing for the patient described in the case, "...important reasons for testing...would include (1) identifying ...high-risk anatomy and (2) determining prognosis." As noted above, determining prognosis per se is not necessarily knowledge that drives specific differential decisions. Moreover, it is particularly not useful in this setting of a new diagnosis of $\mathrm{HF}$ with systolic dysfunction prior to any treatment, as the prognosis changes dramatically based on the initial response to guideline-directed medical therapy. Those patients with a robust reverse remodeling response to initial medical therapy have a much lower risk subsequent prognosis compared to those with more persistent LV dilatation and dysfunction. $^{13}$

The use of nuclear techniques is discussed by Caobelli and Bengel. ${ }^{14}$ SPECT stress/rest imaging like echocardiography has modest performance for detecting CAD using a standard CAD detection threshold in the setting of $\mathrm{HF}$ but has high negative predictive value to rule out extensive CAD likely related directly to the cardiomyopathic state. ${ }^{15}$ Thus, a normal stress perfusion study in the setting of a dilated, dysfunctional ventricle makes it highly unlikely that the patient has ischemic cardiomyopathy. These authors describe the performance of SPECT and PET for detecting CAD-a do some of the other authors-from the perspective of the larger literature involving patients referred for stress testing for suspected CAD. Those data are not necessarily directly relevant to the issues at hand. Specificity is likely lower, as patients with nonischemic cardiomyopathy can have perfusion defects unrelated to 
epicardial CAD. ${ }^{16}$ Moreover, the question being asked in patients with suspected CAD and chest pain symptoms-is any "obstructive", CAD present causing ischemia and the patient's symptoms-is a somewhat distinct question from the one being asked in the setting of $\mathrm{HF}$ and systolic dysfunction. The presence of CAD in such a heart failure patient often leads to a label of "ischemic cardiomyopathy.", But as alluded to by Danad and Min, ${ }^{7}$ the presence of any stenosis $\geq 50 \%$ identifies a patient as having CAD by traditional definition, but it does not necessarily mean that patient has an ischemic cardiomyopathy. If the patient under discussion, with mild diffuse hypokinesis and an EF of $35 \%$, was found to have a $70 \%$ stenosis of the mid-left circumflex, she would have CAD and a cardiomyopathy, but those 2 entities would not be related. Most specifically, ischemic cardiomyopathy results from either a sizeable infarction with attendant remodeling or from extensive hibernation, or some combination thereof, and is thus best identified by interrogation of the myocardial substrate, with subsequent demonstration of the coronary anatomy.

Certainly, SPECT and PET techniques for assessing viability and potential benefit of revascularization have a solid literature base and have been widely used for that purpose. ${ }^{17}$ A very abnormal perfusion study makes ischemic cardiomyopathy quite likely, and the extent of ischemia or viability (possibly demonstrated by a PET study of glucose metabolism) can identify potential benefit from revascularization.

Caobelli and Bengel also discuss molecular imaging techniques involving radionuclide tracers to "... assist in...key clinical decisions in heart failure', ${ }^{14}$ Imaging sympathetic innervation with agents such as ${ }^{123} \mathrm{I}-$ metaiodobenzylguanidine (mIBG) or ${ }^{11} \mathrm{C}$-hydroxyephedrine is theoretically attractive to potentially assess arrhythmic risk and perhaps guide ICD decisions as they suggest, but none of the data published to date are adequately powered to enable identification of lowrisk patients with sufficient confidence to allow a decision not to implant an ICD in someone who otherwise has a clinical indication, as discussed elsewhere. ${ }^{18}$ Patients with specific HF etiologies may indeed benefit from targeted imaging for syndromes such as sarcoidosis or cardiac amyloidosis, information that is evolving. ${ }^{19}$

The argument for cardiac magnetic resonance imaging is made by Shaw and Kramer. ${ }^{20}$ The use of late gadolinium enhancement imaging in patients with $\mathrm{HF}$ and systolic dysfunction has informed a more nuanced understanding of the differentiation of ischemic vs. nonischemic cardiomyopathy, beyond simply reflecting the status of the coronary anatomy. Beginning with the seminal study of McCrohon et al., ${ }^{16}$ it has become very clear that patients we think of as having nonischemic cardiomyopathy (on the basis of normal or nonobstructive coronary anatomy) may have extensive areas of fibrosis in noncoronary distributions or even coronary vascular distributions and thus may have "ischemic", cardiomyopathy with respect to the mechanism of the myocardial damage and subsequent LV dysfunction. Various etiologies of nonischemic cardiomyopathy can be suggested by the patterns of LGE, such as diffuse subendocardial enhancement in some forms of cardiac amyloid, which would allow specific diagnoses. ${ }^{20}$ Other techniques described by the authors such as T1 mapping, and T2 weighted imaging and mapping that can interrogate features of the myocardial tissue at present are evolving, and whether results lead to specific management strategies is not as yet clear. T2* mapping in the setting of iron-overload cardiomyopathy can indeed be a valuable management tool for monitoring chelation therapy $^{21}$ and can identify myocardial iron for diagnostic purposes.

The technique of assessing stress and rest perfusion with pharmacologic stress and CMR assessment of the first pass of gadolinium has been evaluated in patients with suspected $\mathrm{CAD}^{22}$ It would be theoretically attractive in the setting of $\mathrm{HF}$ and systolic dysfunction based on the data from the SPECT and PET literature. To date, there is little validation of its use in this setting, however, and it is not described by Shaw and Kramer as part of the comprehensive CMR exam of a patient with a new diagnosis of $\mathrm{HF}^{20}$

All of the authors have made passionate cases for their modalities of choice. Where does that leave us with this patient? I think it is fair to say that no one modality at the moment can answer every question we may have in this situation in one sitting. However, approaching the issue from the reductionist perspective of obtaining enough information to make an initial clinical decision, with selective additional imaging conditional on the initial results provides the framework to move ahead.

If we start with CT angiography and find normal coronary arteries, we must then move on to assess primary myocardial etiologies. If we find extensive anatomic CAD, we must then assess ischemia and viability with another test (presuming that most laboratories cannot at this point do CT-based myocardial tissue characterization). Thus, starting with $\mathrm{CT}$ would often lead to the need for a second test.

If we start with stress/rest SPECT or PET imaging and find normal perfusion, again there would be a need to assess primary myocardial etiologies. If there are extensive severe fixed perfusion abnormalities, that might complete our assessment, with a diagnosis of ischemic cardiomyopathy which is unlikely to benefit from revascularization. In that case, there is little need for invasive coronary angiography. Similarly, if there 
are extensive areas of ischemia, ischemic cardiomyopathy is likely and there is potential benefit of revascularization; the next step would be coronary angiography to assess the technical feasibility. Thus, SPECT or PET may provide sufficient information after the initial test to move down a therapeutic path, but sometimes additional information would be needed.

That would also be the case for CMR imaging. The finding of very extensive LGE in a transmural infarct pattern in a coronary vascular distribution would be consistent with a diagnosis of ischemic cardiomyopathy and a patient who is unlikely to benefit from revascularization; thus, no further imaging would be needed. The absence of such a finding, however, leaves open the possibility of extensive hibernation, which may be assessed by stress/rest perfusion imaging by CMR, or by another modality. As noted, other LGE patterns may point to very specific diagnoses.

While the data on the use of stress echocardiography (with dobutamine) to assess contractile reserve for the specific question of regional viability are solid (in the setting of known CAD, regional wall motion abnormality and consideration of revascularization), the general use of stress echocardiography more proximally in the work-up of such a patient may not answer the specific questions at hand with sufficient rigor.

As we think about ordering one or more of our sophisticated imaging modalities, I can hear the voice of Dr. Iskandrian at a case presentation conference reminding us not to forget the simple clinical information. In this case, the mild diffuse wall motion abnormality to some degree argues against an ischemic cardiomyopathy but not strongly enough on its own to rule that out and obviate the need for imaging. The absence of low voltage on ECG makes an infiltrative disease somewhat unlikely. The combination of diabetes and such a cardiomyopathy brings to mind the possibility of iron overload, and certainly a serum test is simpler and less expensive than $\mathrm{T} 2 *$ imaging as a first step!

The use of imaging techniques for patients with heart failure has evolved substantially over the years. We have learned an enormous amount about pathophysiology from imaging the ischemic substrate and the myocardial substrate, knowledge which has allowed us to open up treatment paths for patients. However, in the current era we now need to think carefully about deploying these modalities in an efficient manner, thinking clearly about the proximal questions at hand, gathering enough information to answer them, and only moving on to additional testing when specific questions remain. It is important to avoid the "TMI" (too much information"') syndrome.

\section{Disclossure}

No conflicts relevant to this work.

\section{References}

1. Yancy CW, Jessup M, Bozkurt B, Butler J, Casey DE Jr, Drazner $\mathrm{MH}$ et al. 2013 ACCF/AHA guideline for the management of heart failure: a report of the American College of Cardiology Foundation/American Heart Association Task Force on Practice Guidelines. Circulation 2013;128:e240-327.

2. Felker GM, Fiuzat M, Thompson V, et al. Soluble ST2 in ambulatory patients with heart failure: association with functional capacity and long-term outcomes. Circ Heart Fail 2013;6:1172-9.

3. Felker GM, Fiuzat M, Shaw LK, Clare R, Whellan DJ, Bettari L et al. Galectin-3 in ambulatory patients with heart failure: results from the HF-ACTION study. Circ Heart Fail 2012;5:72-8.

4. Ky B, French B, McCloskey K, Rame JE, McIntosh E, Shahi P et al. High-sensitivity ST2 for prediction of adverse outcomes in chronic heart failure. Circ Heart Fail 2011;4:180-7.

5. Ahn SG, Samady H. How to differentiate the etiology of LV dysfunction as to whether it is "ischemic cardiomyopathy" or "dilated non-ischemic cardiomyopathy"'? invasive coronary and myocardial assessment is the approach of first choice. J Nucl Cardiol 2015. doi:10.1007/s12350-015-0135-2.

6. The ESCAPE Investigators and ESCAPE Study Coordinators. Evaluation study of congestive heart failure and pulmonary artery catheterization effectiveness: the ESCAPE trial. JAMA 2005 294:1625-33

7. Danad I, Min JK. Computed tomography: the optimal imaging method for differentiation of ischemic versus non-ischemic cardiomyopathy. J Nucl Cardiol 2015. doi:10.1007/s12350-015-0146-z.

8. Min JK, Leipsic J, Pencina MJ, et al. Diagnostic accuracy of fractional flow reserve from anatomic CT angiography. JAMA 2012;308:1237-45.

9. Argulian E, Chaudhry FA. Evaluating left ventricular systolic dysfunction: stress echocardiography. J Nucl Cardiol 2015. doi: 10.1007/s12350-015-0116-5.

10. Cornel JH, Bax JJ, Elhendy A, et al. Biphasic response to dobutamine predicts improvement of global left ventricular function after surgical revascularization in patients with stable coronary artery disease: implications of time course of recovery on diagnostic accuracy. J Am Coll Cardiol 1998;31:1002.

11. Mullens W, Borowski AG, Curtin RJ, Thomas JD, Tang WH. Tissue doppler imaging in the estimation of intracardiac filling pressure in decompensated patients with advanced systolic heart failure. Circulation 2009;119:62-70.

12. Santos M, Rivero J, McCullough SD, West E, Opotowski AR, Waxman AB, Systrom DM, Shah AM. E/e' ratio in patients with unexplained dyspnea: lack of accuracy in estimating left ventricular filling pressure. Circ Heart Fail 2015. doi:10.1161/ CIRCHEARTFAILURE.115.002161

13. Merlo M, Pyxaras SA, Pinamonti B, Barbati G, Di Lenarda A, Sinagra G. Prevalence and prognostic significance of left ventricular reverse remodeling in dilated cardiomyopathy receiving tailored medical treatment. J Am Coll Cardiol 2011;57:1468-76.

14. Caobelli F, Bengel FM. Ischaemic vs non-ischaemic dilated cardiomyopathy: the value of nuclear cardiology techniques. J Nucl Cardiol 2015. doi:10.1007/s12350-015-0128-1.

15. Soman P, Lahiri A, Mieres JH, Calnon DA, Wolinsky D, Beller GA et al. Etiology and pathophysiology of new-onset heart failure: evaluation by myocardial perfusion imaging. J Nucl Cardiol 2009;16:82-91. 
16. McCrohon JA, Moon JC, Prasad SK, McKenna WJ, Lorenz CH, Coats AJ et al. Differentiation of heart failure related to dilated cardiomyopathy and coronary artery disease using gadoliniumenhanced cardiovascular magnetic resonance. Circulation 2003; 108:54-9.

17. Allman KC, Shaw LJ, Hachamovitch R, Udelson JE. Myocardial viability testing and impact of revascularization on prognosis in patients with coronary artery disease and left ventricular dysfunction: a meta-analysis. J Am Coll Cardiol 2002;39:1151-8.

18. Wessler BS, Udelson JE. Neuronal dysfunction and medical therapy in heart failure: can an imaging biomarker help to "personalize" therapy? J Nucl Med 2015;56:20S-4S.
19. Udelson JE. Developing imaging biomarkers for myocardial involvement in amyloidosis: challenge and opportunity. JACC Cardiovasc Imaging 2015;8:60-2.

20. Shaw PW, Kramer CM. The case for CMR. J Nucl Cardiol 2015. doi:10.1007/s12350-015-0147-y.

21. Kirk P, Roughton M, Porter JB, Walker JM, Tanner MA, Patel J, Wu D, Taylor J, Westwood MA, Anderson LJ, Pennell DJ. Cardiac $\mathrm{T} 2 *$ magnetic resonance for prediction of cardiac complications in thalassemia major. Circulation 2009;120:1961-8.

22. Heydari B, Jerosch-Herold M, Kwong RY. Assessment of myocardial ischemia with cardiovascular magnetic resonance. Prog Cardiovasc Dis 2011;54:191-203. 